\title{
Some Applications of Ricci Flow in Physics
}

\author{
E Woolgar 1 \\ Dept of Mathematical and Statistical Sciences, University of Alberta, \\ Edmonton, AB, Canada T6G 2G1. \\ Dedicated to Rafael D Sorkin on the occasion of his 60th birthday. \\ Based on a keynote talk given at the Theory Canada III conference, \\ Edmonton, June 2007.
}

\begin{abstract}
I discuss certain applications of the Ricci flow in physics. I first review how it arises in the renormalization group (RG) flow of a nonlinear sigma model. I then review the concept of a Ricci soliton and recall how a soliton was used to discuss the RG flow of mass in 2-dimensions. I then present recent results obtained with Oliynyk on the flow of mass in higher dimensions. The final section discusses how Ricci flow may arise in general relativity, particularly for static metrics.
\end{abstract}

\footnotetext{
${ }^{1}$ ewoolgar@math.ualberta.ca
} 


\section{Introduction}

The Ricci flow

$$
\frac{\partial g_{i j}}{\partial \lambda}=-2 R_{i j}
$$

was introduced by Hamilton [1] over 25 years ago. During the intervening time, it has been studied by mathematicians primarily as a tool for proving the geometrization conjecture for closed 3-manifolds, which includes as a special case the Poincaré conjecture that every closed, simply connected 3-manifold is a 3-sphere. This endeavor has now met with success [2, 3, 4].

The equation describes the deformation of a Riemannian metric $g_{i j}$ with "time" $\lambda$. The deformation is driven by Ricci curvature, so that parts of the manifold with greater Ricci curvature will undergo greater deformation. Regions with very little Ricci curvature will change only a little during the flow. Fixed points of the flow are the Ricci flat manifolds

$$
R_{i j}=0
$$

At about the time of Hamilton's work, equation (I.1) was already making its first appearance in physics in Friedan's 1980 thesis [5] on the renormalization group flow of nonlinear sigma models. Equation (I.1), with the coefficient 2 on the right-hand side replaced by a positive constant written as $T$ by Friedan but now denoted by $\alpha^{\prime}$, was the renormalization group (RG) flow equation with the $\beta$-function truncated to leading order in $\alpha^{\prime}$.

The mathematical study of the Ricci flow has experienced tremendous growth in the aftermath of Perelman's breakthrough work [2] with its consequences for geometrization. It is a good time to look for physical applications. There are several possibilities, some but not all related to renormalization group flow. A brief list of Ricci flow questions and their possible physical applications would include:

- Are there classes of Lorentzian metrics to which Ricci flow can be applied? (I say "classes" in part because the unrestricted initial value problem would not be well-posed.) If so, this would surely find application in general relativity, but what precisely are the useful applications?

- Is flat space stable under Ricci flow? If not, then mass would behave in unexpected ways under RG flow. This would have applications to tachyon condensation in string theory. 
- Can we do Ricci flow on manifolds with boundary? There is recent work on this in the context of an application to black hole thermodynamics [6]. As well, this question has some relevance to certain formulations of quasilocal mass.

- Can Perelman's entropy definition be generalized to full RG flow (of nonlinear sigma models) to express this as a gradient flow on a space of coupling constants equipped with a positive definite metric? If so, then this would yield a $C$-function for this flow. See [7, 8].

This list is not intended to be exhaustive by any means, but is already too large to be addressed in any reasonably complete fashion in the present article. I will confine myself to an introductory treatment of Ricci flow for physicists working in general relativity and quantum field theory, together with a report on some recent progress in two areas: the flow of mass and the flow of static metrics.

Section II has a brief discussion of Ricci flow as an approximation to RG flow for Sigma models. Section III introduces the important notion of self-similar Ricci flow solutions called Ricci solitons. Section IV reviews a discussion in [9], which uses a 2-dimensional Ricci soliton to illustrate the behaviour of mass under Ricci flow. Section V describes recent results that Oliynyk and I have obtained regarding the mass question in dimension 3 and greater, in spherical symmetry [10]. Section VI considers the Ricci flow of static spacetime metrics and describes a soliton recently found by Akbar and me [11] which can be interpreted as generating a nontrivial Lorentzian flow.

Acknowledgements. This work was partially supported by an NSERC Discovery Grant to the author, who wishes to thank the organizers for the opportunity to speak about this work at Theory Canada III. I thank my collaborators M Akbar, T Oliynyk, and V Suneeta for discussions and for permitting me to draw on our joint work herein.

I first came to appreciate the importance for physics of a firm mathematical foundation from Rafael Sorkin. Early on, together with Penrose, we formulated an argument for a positive mass theorem which drew on causal structure of Lorentzian manifolds but also relied heavily on the kinds of arguments that arise in both comparison geometry and differential topology. What I learned then has influenced me ever since, as will I hope be at least somewhat evident in what follows below. It is a pleasure to dedicate this article to Rafael on the occasion of his 60th birthday. 


\section{Ricci flow and Sigma Models}

Let us start with the connection between Ricci flow and RG flow for string theory or, more precisely, for bosonic nonlinear sigma models from 2-dimensional spacetime to a curved Riemannian target manifold. Consider the sigma model with only the coupling to the target space metric $g_{i j}$ (it's easy to add a dilaton). The action is

$$
S=\alpha^{\prime} \int_{M} h^{a b}(\sigma) g_{i j}(X) \frac{\partial X^{i}}{\partial \sigma^{a}} \frac{\partial X^{j}}{\partial \sigma^{b}} \sqrt{h} d^{2} \sigma
$$

plus a boundary term if the manifold $M$ is not closed. Here $\sigma=\left(\sigma^{1}, \sigma^{2}\right)$ denotes coordinates on the worldsheet, $h_{a b}$ is any metric on that worldsheet, $X^{i}$ denotes the target manifold coordinates of the embedded (or immersed) worldsheet, and $\alpha^{\prime}$ is a square distance (the string scale). Thinking of this as the starting point for a theory of quantum scalar fields $X^{i}$ in 2 dimensions, then $g_{i j}(X)$ represents the coupling constants of the theory and can be expected to renormalize. The renormalization group flow can be expressed perturbatively as a power series in $\alpha^{\prime}[5]$ :

$$
\frac{\partial g_{i j}}{\partial \lambda}=-\alpha^{\prime} R_{i j}-\frac{\alpha^{\prime 2}}{2} R_{i k l m} R_{j}^{k l m}+\ldots
$$

where the ellipsis denotes a power series with terms of order $\alpha^{\prime 3}$ and higher.

Now say that $g(\lambda, \cdot)$ is a solution of this equation on a "time" (that is, energy) interval $\lambda \in\left(T_{1}, T_{2}\right)$. Define

$$
\begin{aligned}
\hat{\lambda} & :=a \lambda \in\left(\hat{T}_{1}, \hat{T}_{2}\right):=\left(a T_{1}, a T_{2}\right), \\
\hat{g}_{i j}(\hat{\lambda}, \cdot) & :=a g_{i j}(\hat{\lambda} / a, \cdot) \equiv a g_{i j}(\lambda, \cdot),
\end{aligned}
$$

for some constant $a>0$. The RG flow equation becomes

$$
\frac{\partial \hat{g}_{i j}}{\partial \hat{\lambda}}=-\alpha^{\prime} \hat{R}_{i j}-a \frac{\alpha^{\prime 2}}{2} \hat{R}_{i k l m} \hat{R}_{j}^{k l m}+\ldots
$$

The terms represented by the ellipsis have coefficients $a^{p-1} \alpha^{\prime p}$ with $p \geq 3$. Thus, if we take $a \rightarrow 0$, we obtain the Ricci flow equation (I.1).

However, this does not necessarily mean that solutions of (I.1) and (II.4) approach each other on arbitrary $\lambda$ scales as $a$ becomes small. Even worse, if the original solution $g(\lambda, p)$ was defined on a bounded interval $\lambda \in\left(T_{1}, T_{2}\right)$, then rescaling shrinks this interval to $\hat{\lambda} \in\left(a T_{1}, a T_{2}\right)$, which tends to zero width. To avoid this, we will primarily consider solutions $g$ defined on 
a semi-infinite or infinite domain, so for any finite $a$ the domain of $\hat{\lambda}$ is also semi-infinite or infinite. Flows on such domains are classified as either ancient $(\lambda \in(-\infty, 0))$, immortal $(\lambda \in(0, \infty))$, or eternal $(\lambda \in(-\infty, \infty))$. The ancient and eternal flows are of greatest interest. If an ancient or eternal Ricci flow stays close to a full RG flow as $\lambda \rightarrow-\infty$, then the sigma model is well-defined when the energy cutoff is removed 2

By theorem (9.15) of [12, if an ancient or eternal solution $\hat{g}_{i j}(\hat{t})$ is asymptotically flat at each $\hat{t}$ (so the sectional curvatures are always bounded), then the scalar curvature obeys $R \geq 0$ for all $\hat{t}$. That means the positive mass theorem is obeyed all along the flow, and so we learn that positive target manifold mass is a requirement for a well-defined sigma model at high energy.

This also provokes the question of how mass behaves under RG flow or at least, in the current context, under Ricci flow. This leads us first to a discussion of a special solution of the flow known as a Ricci soliton, and then to the question of whether flat space is asymptotically stable with respect to Ricci flow.

\section{Solitons}

As a model for Ricci flow, consider the one-dimensional linear heat equation

$$
\frac{\partial u}{\partial t}=\frac{\partial^{2} u}{\partial x^{2}}
$$

for the temperature $u(t, x)$.

For the moment, let $x$ take values in $[0, \pi]$ and impose periodic boundary conditions $u(t, 0)=u(t, \pi)=0$. Then there are separable solutions

$$
u_{n}(t, x)=T_{n}(t) X_{n}(x)=e^{-n^{2} t} \sin n x
$$

\footnotetext{
${ }^{2}$ Think of a quantum field theory characterized by $k$ coupling constants $c_{i}$, all of whose UV divergences can be traced back to just one particular Feynman graph. Impose a momentum cutoff $\Lambda$, so the graph takes a finite value $\Gamma\left(\Lambda, c_{1}, \ldots, c_{k}\right)=C=$ const. The predictions of the theory will now depend on the cut-off $\Lambda$ as well as on the coupling constants $c_{i}$. Now follow a curve on the level set $\Gamma=C$, along which the coupling constants $c_{i}$ and the cut-off $\Lambda$ will vary but of course $\Gamma$ will not. This is an RG flow. If one can follow some such curve until $\Lambda \rightarrow \infty$, introducing no new divergences in other graphs, this is cutoff removal. The limiting theory (defined by coupling constants $c_{i}(\infty)$ ) obviously has no $\Lambda$ dependence and its Feynman diagrams have no UV divergences. This can work even when more than one Feynman graph is responsible for the divergences of the theory, provided there are sufficiently many coupling constants.
} 
for $n$ a positive integer. Since the equation is linear, then arbitrary linear combinations

$$
u(t, x)=\sum_{n=1}^{\infty} c_{n} e^{-n^{2} t} \sin n x
$$

are also solutions, where the $c_{n}$ are constants. An elementary argument using the maximum principle shows that this is the general solution. The Fourier decomposition of the initial data $u(0, x)$ uniquely determines the constants $c_{n}$.

Separable solutions (III.2) are self-similar. Self-similarity means that they evolve in time purely by rescaling the amplitude. Now the general solution is not self-similar because it is not separable. Self-similar solutions arise only for very special initial data. Specifically, if the initial data contain precisely one Fourier mode, say the $n^{\text {th }}$ mode, then the particular solution evolving from that data is $u_{n}(t, x)=c_{n} e^{-n^{2} t} \sin n x$ and is self-similar and separable.

If the initial data contain many Fourier modes, the lowest of which is the $n^{\text {th }}$ mode, then at late times the higher modes $m>n$ are suppressed relative to this mode by a factor of $e^{-\left(m^{2}-n^{2}\right) t}$, so the solution tends to $e^{-n^{2} t} \sin n x$. Solutions always approach self-similarity at late times.

In Ricci flow, self-similar solutions exist and are called Ricci solitons. They are always either anceint, immortal, or eternal, and so are of interest for us. Of course, in contrast to the heat equation case, an arbitrary Ricci flow is not a sum of solitons, since Ricci flow is nonlinear, but general flows sometimes asymptote to solitons in certain limits.

Steady solitons do not rescale under Ricci flow. Any Ricci-flat metric is an example of a steady soliton. There are also expanding and shrinking solitons, which do rescale. A simple example of a shrinking soliton is the round shrinking $n$-sphere

$$
g_{i j}(\lambda)=2(n-1)(\Lambda-\lambda) g_{i j}^{\text {can }}, \Lambda=\text { const },
$$

where $g^{\text {can }}$ denotes the canonical sphere metric $d \theta_{1}^{2}+\sin ^{2} \theta_{1}\left(d \theta_{2}^{2}+\ldots\right)$. This solution exists for $\lambda \in(-\infty, \Lambda)$ and is said to "extinguish" at $\lambda=\Lambda$. Like the lowest Fourier mode in heat flow, this soliton "attracts" in the sense that any slightly deformed sphere will approach the round shrinking sphere as flows toward extinction.

A more sophisticated example is the solvegeometry expanding soliton (Bianchi type $\mathrm{IV}_{-1}$ )

$$
d s^{2}=e^{-2 z} d x^{2}+e^{2 z} d y^{2}+4 \lambda d z^{2} .
$$


This metric has a discrete group of isometries such that the quotient space is a smooth compact manifold [13], but the soliton interpretation is valid only on $\Re^{3}$, not on a quotient.

Why is this a Ricci soliton? To answer, we can directly compute the Ricci curvature of (III.5), and we get $R_{i j}=-2 \delta_{i}^{z} \delta_{j}^{z}$. Also by direct computation, we see that $\frac{\partial g_{i j}}{\partial \lambda}=4 \delta_{i}^{z} \delta_{j}^{z}$. Comparing these two results, we see that (I.1) is satisfied. So we have a Ricci flow, but is it self-similar? To answer this, we change coordinates and write the metric (III.5) as

$$
d s^{2}=g_{i j}(\tilde{x}) d \tilde{x}^{i} d \tilde{x}^{j}=4 \lambda\left(e^{-2 \tilde{z}} d \tilde{x}^{2}+e^{2 \tilde{z}} d \tilde{y}^{2}+d \tilde{z}^{2}\right)=4 \lambda g_{i j}^{0}(\tilde{x}) d \tilde{x}^{i} d \tilde{x}^{j},
$$

where we have performed a $\lambda$-dependent diffeomorphism $\phi_{\lambda}$ taking $x \mapsto \tilde{x}=$ $\frac{x}{\sqrt{4 \lambda}}, y \mapsto \tilde{y}=\frac{y}{\sqrt{4 \lambda}}$, and $z \mapsto \tilde{z}=z$. Since the coefficients $g_{i j}^{0}$ do not vary with $\lambda, d s^{2}$ evolves self-similarly; i.e., it evolves only through the overall scale factor $4 \lambda$ in front of $g^{0}$ and via the $\lambda$-dependent diffeomorphism $\phi_{\lambda}$.

However, now we have from (III.6) that $\frac{\partial g_{i j}}{\partial \lambda}=g_{i j}^{0}$, while the Ricci curvature has not changed, and so now $\frac{\partial g_{i j}}{\partial \lambda} \neq-2 R_{i j}$ ! This shows that (I.1) is not geometrical, in that it depends on the choice of coordinates along the flow. There should really be an extra Lie derivative term accounting for the fact that the coordinates can vary with $\lambda$. This is a new feature not seen in our scalar 1-dimensional heat equation analogy, and gives rise to the Hamilton-DeTurck equation

$$
\frac{\partial g_{i j}}{\partial \lambda}=-2 R_{i j}+£_{X} g_{i j}
$$

where the vector field $X$ generates the diffeomorphism $\phi_{\lambda}$ by which the coordinates vary along the flow and $£_{X}$ denotes the Lie derivative along $X$. This also gives rise to the Ricci soliton equation

$$
R_{i j}-\frac{1}{2} £_{X} g_{i j}-c g_{i j}=0,
$$

where $c$ is a constant. If $g$ is a solution of this equation, then there is a Ricci flow given by $a(\lambda) \phi_{\lambda}^{*} g$, and so this flow is self-similar. For the solvegeometry, the metric $g^{0}$ is a solution of (III.8).

\section{The Mass of a 2-Dimensional Soliton}

We now begin our discussion of the behaviour of mass under Ricci flow. Before passing to the asymptotically flat case, this section will discuss the 
2-dimensional, rotationally symmetric, expanding soliton used in a study of mass under RG flow [9]. This soliton is immortal but not eternal. It's a gradient soliton, meaning that the vector field $X$ generating the soliton is the gradient of a scalar potential $X^{i}=g^{i j} \nabla_{j} \varphi$. The metric and potential function can be written as

$$
\begin{aligned}
d s^{2} & =\lambda\left(f^{2}(r) d r^{2}+r^{2} d \theta^{2}\right), \\
\varphi(r) & =\int r^{\prime} f\left(r^{\prime}\right) d r^{\prime},
\end{aligned}
$$

where $f$ is given implicitly by

$$
\left(\frac{1}{\zeta}-1\right) \exp \left(\frac{1}{\zeta}-1-\frac{x^{2}}{2 \alpha^{\prime}}\right)=\left(\frac{1}{f(x)}-1\right) \exp \left(\frac{1}{f(x)}-1\right),
$$

where $\zeta=$ const. What is important is that $f(x) \rightarrow \zeta$ for $x \rightarrow 0^{+}, f(x) \rightarrow 1$ for $x \rightarrow \infty$, and $f$ is monotonic in between. Changing coordinates to $\rho=$ $r \sqrt{\lambda}$, we get

$$
d s^{2}=f^{2}(\rho / \sqrt{\lambda}) d \rho^{2}+\rho^{2} d \theta^{2} .
$$

Now take $\theta \in[0,2 \pi \zeta]$. For $\lambda \rightarrow 0^{+}$, at any fixed $\rho>0$ (and thus at any fixed proper distance from the origin), we have $f(\rho / \sqrt{\lambda}) \rightarrow 1$. The limiting metric (in the Gromov-Hausdorff sense: at each $\lambda$ we fix a point, here the origin, and study the metric within arbitrarily large proper radius balls about this point) is a flat cone of deficit angle $\delta=2 \pi(1-\zeta)$. This is the special initial condition which gives rise to this soliton. Now in 2 dimensions, deficit angle plays the role of mass, so $2 \pi(1-\zeta)$ is the mass.

Since this is an expanding soliton, one might at first expect the mass $\delta$ to rescale with $\lambda$ but this is not what happens. At any fixed $\lambda>0$, the metric is asymptotic at large $\rho$ to the same flat cone, so the mass remains the same. But at any $\lambda>0$, there is no cone point (since $f(\rho / \sqrt{\lambda}) \rightarrow \zeta$ for $\rho \rightarrow 0$ with $\lambda>0$ fixed), so the manifold is smooth.

However, as $\lambda \rightarrow \infty$, the curvature within any fixed distance of the origin dissipates and so the Gromov-Hausdorff limit is just flat space. Thus, if the initial $\lambda=0$ flat cone has a positive deficit angle (i.e., if $0<\zeta<1$ ), the mass "decreases" in this sense to a flat and thus zero mass metric in the infinite time limit; if the initial deficit angle is negative (i.e., if $\zeta>1$ ), the mass increases to zero in this sense 3 Either way, the flow is attracted to flat space in the limit of large $\lambda$, but at any finite $\lambda$ the mass (=deficit angle) is unchanged from its initial value.

\footnotetext{
${ }^{3}$ Smoothness at the origin during the flow is actually a boundary condition imposed at
} 


\section{Mass in Higher Dimensions}

In dimension $n \geq 3$, we can define asymptotic flatness by choosing suitable coordinates near infinity which will be Cartesian coordinates for a flat reference metric. An asymptotically flat metric is one whose components differ from the reference by an element of some $H_{\delta}^{k}$ space, where the fall-off rate $\delta$ (not related to $\delta$ in the previous section) is sufficient to give a well-defined ADM mass. For defintions and details, see [10].

Returning momentarily to the heat equation analogy, recall that the 1dimensional heat equation on an infinite domain has a certain non-uniqueness. Namely, Tychonoff pointed out that

$$
u(t, x)=\sum_{k=0}^{\infty} \frac{x^{2 k}}{(2 k) !} \frac{d^{k}}{d t^{k}} e^{-1 / t^{2}}
$$

solves the 1-dimensional heat equation for $t \in(0, \infty)$ and $\lim _{t \rightarrow 0^{+}} u(t, x)=$ 0 . Thus, we can add an arbitrary multiple of (V.1) to any solution to get a new solution for the same initial data. An extreme case is if the temperature is initially zero everywhere, but at any positive time the temperature distribution can be that described by (V.1), and so is large at large $x$.

This shows that it is necessary to impose fall-off conditions on solutions of the heat equation all along the flow, not just on the initial temperature distribution, in order to have uniqueness. Remarkably, this problem does not seem to arise for Ricci flow. At least, asymptotic flatness of initial data is always preserved so long as the flow remains nonsingular [18, 19]. Not only that, but the mass does not change, just as we saw in the rotationally symmetric 2-dimensional example of the last section, and as we will now see in higher dimensions (without assuming any symmetry).

Because asymptotic flatness is preserved, we can evaluate the mass at any time along the flow. If the mass evaluated at two different $\lambda$-values differs, then at some intermediate $\lambda$-value the mass must have nonzero flow derivative. But the following simple computation shows that this cannot happen.

On an asymptotic end $U \subseteq M$, let $\delta_{a b}$ be a fixed (constant along the flow) Euclidean metric compatible with the derivative $\partial_{a}$ and let $\Sigma$ be a closed embedded hypersphere. Define the functional $E_{\Sigma}: S^{2} U \rightarrow \Re$ acting

the origin, where the polar coordinate system breaks down. One can impose any deficit angle one wishes at the origin instead. Then the $\lambda \rightarrow \infty$ limiting metric will be a flat cone with this deficit angle. Therefore, this solution can describe transitions between two different flat cones. 
on symmetric covariant 2-tensors by

$$
E_{\Sigma}(g):=\frac{1}{16 \pi} \int_{\Sigma}\left[\eta^{i} \partial^{j} g_{i j}-\eta \cdot \partial\left(\delta^{i j} g_{i j}\right)\right] d A(\delta)
$$

where $\partial^{i}:=\delta^{i j} \partial_{j}$ and $\eta$ is the outward unit normal vector to $\Sigma$ wrt the metric $\delta$. If $\Sigma$ belongs to a sequence of hyperspheres $\Sigma_{n}$ whose interiors exhaust $U$, then in the limit we get the $\operatorname{ADM}$ mass of $g$ :

$$
E^{\mathrm{ADM}}=\lim _{n \rightarrow \infty} E_{\Sigma_{n}}(g)
$$

Now this functional is linear in $g$ so

$$
\frac{d E_{\Sigma}}{d t}=E_{\Sigma}\left(\frac{\partial g}{\partial t}\right)=\frac{1}{16 \pi} \int_{\Sigma}\left[\eta^{i} \partial^{j} \frac{\partial g_{i j}}{\partial t}-\eta \cdot \partial\left(\delta^{i j} \frac{\partial g_{i j}}{\partial t}\right)\right] d A(\delta) .
$$

If the metric evolves by Ricci flow (I.1) then we get

$$
\begin{aligned}
\frac{d E_{\Sigma}}{d t} & =E_{\Sigma}\left(\frac{\partial g}{\partial t}\right)=-\frac{1}{8 \pi} \int_{\Sigma}\left[\eta^{i} \partial^{j} R_{i j}-\eta \cdot \partial\left(\delta^{i j} R_{i j}\right)\right] d A(\delta) \\
& =\frac{1}{8 \pi} \int_{\Sigma} d A(g)\left\{\frac{1}{2} \eta \cdot \nabla R+\mathcal{O}\left(1 / r^{n}\right)\right\} \in \mathcal{O}\left(1 / r^{2}\right) \rightarrow 0
\end{aligned}
$$

where we used the Bianchi identity to pass from one line to the next and used asymptotic flatness to treat derivatives compatible with $\delta$ and $g$ as equivalent near infinity (they differ by terms that fall off fast enough to ignore). We can write the asymptotic flatness condition in the succinct form $\partial^{k}(g-\delta) \in \mathcal{O}\left(1 / r^{n+k-2}\right)$ for $k=0,1,2,3$. This gives immediately that $\nabla R \sim \partial^{3} g \sim 1 / r^{n+1}$ while $d A \sim r^{n-1}$, so the integral falls off as claimed in the last step of (V.5). Hence the derivative of the mass is always zero and so the mass is constant, just as in the special 2-dimensional example of the last section.

Another feature of the 2-dimensional soliton appears relevant in higher dimensions. For rotationally symmetric metrics, the flow converges to flat space [10]. This result assumes the initial data for the flow are asymptotically flat and contain no minimal hypersphere (if such a sphere were present, it might pinch off), but the data are otherwise quite arbitrary. Such a flow is then immortal and (Cheeger-Gromoll) converges to the flat metric in the limit of infinite flow time. Moreover, if the initial metric is not spherically symmetric but is in a certain sense close to being flat, the flow is also immortal and converges to a flat metric [20]. (The notion of "close to flat" 
used in [20] is too restrictive to allow nonzero mass in 3 dimensions, but allows it in all higher dimensions.)

It may seem paradoxical that ADM mass is constant throughout the flow and yet the limiting metric is flat. Certainly then $\lim _{\lambda \rightarrow \infty} E_{\mathrm{ADM}}(g(\lambda)) \neq$ $E_{\mathrm{ADM}}(g(\infty))=0$. The resolution is that the notion of convergence of manifolds in the $\lambda \rightarrow \infty$ limit involves picking a marked point (e.g., the origin of symmetry in the rotationally symmetric case) and examining the geometry of sequences of arbitrarily large metric balls about that point. Thus, the quasilocal mass of these balls is relevant. Oliynyk and I studied the decay of quasilocal mass in our rotationally symmetric flow [10] and found that within any ball of fixed (proper) radius, the quasilocal mass evaporates at a rate $\sim 1 / \lambda$. The coefficient of this rate depends on the radius and vanishes at infinity fast enough that the ADM mass remains constant at any finite $\lambda$.

\section{Static Lorentzian Metrics}

The discussion of the previous section points to the likely importance that an understanding of the flow of quasilocal mass would have for the general question of stability of flat space (and other asymptotically flat fixed points) when no symmetry is assumed. Specifically, an understanding of the conditions under which the mass evaporates monotonically in $\lambda$ is likely to be an important tool.

Conversely, the Ricci flow (and the closely related flow below) may lead to better understanding of quasilocal mass. Bartnik has proposed a definition of quasi-local mass; for details, see 14. Say that $B$ is a Riemannian manifold 4 with boundary $\partial B$. Embed $B$ in an asymptotically flat manifold $(M, g)$ that has non-negative scalar curvature and contains no minimal surface other than possibly a so-called compact outermost horizon. Compute the ADM mass of $(M, g)$. The infimum of the ADM mass over all such possible extensions $(M, g)$ is the Bartnik quasilocal mass of $B$.

Quasilocal mass is important only insofar as it provides a useful geometric characterization of bounded regions within a manifold. As such, the quasilocal mass must have useful properties, among which monotonicity (if $B_{1} \subseteq B_{2}$, then $\left.m_{B_{1}} \leq m_{B_{2}}\right)$ and positivity are classic examples [15, 16]. Bartnik's mass has both of these. But is it nonzero in general? Yes, if the infimum in the definition were a genuine minimum. That is, if there were a specific asymptotically flat but nonflat $R \geq 0$ extension $(M, g) \supset B$ whose $\mathrm{ADM}$ mass were $\leq$ that of any other extension, then this ADM mass and,

\footnotetext{
${ }^{4}$ This is the "spatial" version of the problem. There is also a "spacetime" version.
} 
thus, the Bartnik mass of $B$, would necessarily be $>0$ (by the positive mass theorem).

Bartnik [14] conjectured that a mass-minimizing extension exists and outside of $B$ is a solution of the static Einstein equations. These equations are

$$
\begin{aligned}
R_{i j} & =\nabla_{i} u \nabla_{j} u \\
\Delta u & =0
\end{aligned}
$$

where the second equation is merely an integrability condition for the first; it follows by applying a divergence to (VI.1) and using the contracted second Bianchi identity. Given a solution $(u, g)$ of the fixed point equations, then the metric

$$
d s^{2} \equiv G_{\mu \nu} d x^{\mu} d x^{\nu}=-e^{2 u} d t^{2}+e^{-\frac{2 u}{\sqrt{(n-1)(n-2)}}} g_{i j} d x^{i} d x^{j}
$$

is Ricci-flat and static.

But how does one produce a mass-minimizing metric on the extension of $B$ ? One suggestion is to extend $B$ by an arbitrary asymptotically flat metric obeying "geometric boundary conditions" (that the induced metric and mean curvature of $\partial B$ should match from both sides) and having $R \geq 0$, and then to flow this metric. The problem then is to find a flow which exists subject to these boundary conditions, preserves $R \geq 0$ and asymptotic flatness, and whose fixed points are solutions of (VI.1). If this flow converges to a fixed point, then perhaps we would obtain sequences of metrics converging on a possible minimizer (though if the flow is similar to Ricci flow, we expect the ADM mass to remain constant along the sequence and "jump" at the limit).

Such a flow was studied by List [18] in his $\mathrm{PhD}$ dissertation under the direction of $\mathrm{G}$ Huisken. The flow equations are

$$
\begin{aligned}
\frac{\partial g_{i j}}{\partial \lambda} & =-2\left(R_{i j}-\nabla_{i} u \nabla_{j} u\right) \\
\frac{\partial u}{\partial \lambda} & =\Delta u
\end{aligned}
$$

For reasonable fall-off (say $\partial u \sim 1 / r, \partial^{2} u \sim 1 / r^{2}$ ), ADM mass remains constant along this flow, just as it does for Ricci flow.

If $(u, g)$ satisfies the flow equations (VI.4, VI.5) then the metric

$$
d s^{2} \equiv g_{\mu \nu} d x^{\mu} d x^{\nu}= \pm e^{2 u} d t^{2}+g_{i j} d x^{i} d x^{j}
$$


solves the Hamilton-DeTurck flow

$$
\frac{\partial g_{\mu \nu}}{\partial \lambda}=-2 R_{\mu \nu}+£_{-\nabla u} g_{\mu \nu}
$$

with diffeomorphism generated by $-\nabla u$, where $R_{\mu \nu}$ refers to the metric $g_{\mu \nu}$ of (VI.6). This opens up some possibilities. For example, if we take $g_{i j}$ to have Lorentizian signature, then (VI.1) is the Einstein equation in the presence of a free (massless) scalar field. Many solutions are known (e.g., 21, 22]). Taken together with the "+" sign in (VI.6), this produces a Lorentzian Ricci soliton 5

If $g_{i j}$ has Euclidean signature, non-negative Ricci curvature (as implied by (VI.1) ) is an obstruction to finding nonconstant bounded-below (or above) solutions of (VI.2) [24]. Then $u$ will be unbounded above and below, which is not unacceptable, but a minor modification will circumvent this in any case. Specifically, Akbar and I [11] consider instead the Einstein-free scalar system with a cosmological term

$$
R_{i j}=\nabla_{i} u \nabla_{j} u+\kappa g_{i j},
$$

where we will take $\kappa=$ const $<0$ to make the Ricci curvature nonpositive. As before, (VI.2) is an integrability condition for this equation as well. Then it's straightforward (see [11]) to show that

Lemma. The metric (VI.6) constructed from any solution $\left(u, g_{i j}\right)$ of (VI.8) obeys

$$
R_{\mu \nu}-\frac{1}{2} £_{X} g_{\mu \nu}-\kappa g_{\mu \nu}=0
$$

with $X=-\kappa t \frac{\partial}{\partial t}-g^{i j} \nabla_{i} u \frac{\partial}{\partial x^{j}}$, and is therefore a Ricci soliton.

One example is given by the metric

$$
g_{i j} d x^{i} d x^{j}=d x^{2}+\left(1+e^{\sqrt{2} x}\right)\left(d \theta^{2}+\sinh ^{2} \theta d \phi^{2}\right)
$$

on either $M=\Re^{3}$ or $M=\Re \times \mathcal{H}(g)$ with $\mathcal{H}(g)$ a compact Riemann surface of genus $g>1$. The scalar field is

$$
u=x-\frac{1}{\sqrt{2}} \log \left(1+e^{\sqrt{2} x}\right) .
$$

\footnotetext{
${ }^{5}$ It will be only a local soliton: the singularities of Einstein theory will be present of course; see 23] for a singularity theorem tailored to this.
} 
Clearly this metric is complete and $u$ is everywhere defined on $M$. The corresponding soliton metric

$$
d s^{2}= \pm \frac{e^{2 x}}{\left(1+e^{\sqrt{2} x}\right)^{\sqrt{2}}} d t^{2}+d x^{2}+\left(1+e^{\sqrt{2} x}\right)\left(d \theta^{2}+\sinh ^{2} \theta d \phi^{2}\right)
$$

solves (VI.9) with $\kappa=-1$. It is complete and has bounded sectional curvature. This produces a nontrivial immortal Ricci flow on a nonsingular Lorentzian manifold.

On the issue of the behaviour of mass under the flow (VI.4, VI.5) (particularly, in the limit of approach to fixed points), work continues.

\section{References}

[1] R Hamilton, J Diff Geom 17 (1982) 255.

[2] G Perelman, preprint arxiv:math.DG/0211159]; preprint arxiv:math.DG/0303109.

[3] J Morgan and G Tian, preprint arxiv:math.DG/0607607.

[4] H-D Cao and X-P Zhu, Asian J Math 10 (2006) 165 arxiv:math.DG/0612069].

[5] DH Friedan, PhD thesis, Berkeley (1980), unpublished; Phys Rev Lett 45 (1980) 1057; Ann Phys (NY) 163 (1985) 318.

[6] M Headrick and T Wiseman, Class Quantum Gravit 23(2006) 6683; G Holzegel, T Schmelzer, and C Warnick, preprint arXiv:0706.1694; J Samuel and SR Chowdhury, Class Quantum Gravit 24 (2007) F47.

[7] AA Tseytlin, Phys Rev D75 (2007) 064024 arXiv:hep-th/0612296.

[8] T Oliynyk, V Suneeta, and E Woolgar, Phys Rev D76 (2007) 045001; Nucl Phys B739 (2006) 441 hep-th/0510239]. arXiv:0705.0827];

[9] M Gutperle, M Headrick, S Minwalla, and V Schomerus, JHEP 0301 (2003) 073 arxiv:hep-th/0211063.

[10] T Oliynyk and E Woolgar, Commun Anal Geom, to appear.

[11] M Akbar and E Woolgar, in preparation. 
[12] B Chow and D Knopf, The Ricci Flow: An Introduction, (AMS, Providence, 2004).

[13] WP Thurston, Three-Dimensional Geometry and Topology, ed Silvio Levy (Princeton University Press, 1997).

[14] R Barknik, Proc Int Congress Math, Beijing 2002, vol 2, 231 arXiv:math/0304259]; Tsing Hua Lectures on Geometry and Analysis, ed S-T Yau (International Press, Cambridge MA, USA, 1995) pp $5-27$.

[15] R Geroch, Ann NY Acad Sci 224 (1973) 108.

[16] G Huisken and T Ilmanen, Int Math Res Not 20 (1997) 1045; J Diff Geom 59 (2001) 353.

[17] R Bartnik, private communication.

[18] B List, PhD thesis, Freie Universität Berlin (2005), unpublished.

[19] X Dai and L Ma, preprint [arxiv:math/0510083]; T Oliynyk and E Woolgar, preprint arxiv:math/0607438.

[20] OC Schnürer, F Schulze, and M Simon, preprint arXiv:0706.0421].

[21] AI Janis, ET Newman, and J Winicour, Phys Rev Lett 20 (1968) 878.

[22] M Wyman, Phys Rev D24 (1981) 839.

[23] JE Chase, Commun Math Phys 19 (1970) 276; PhD thesis, University of Alberta (1972), unpublished.

[24] S-T Yau, Commun Pure Appl Math 28 (1975) 201. 